\title{
Ground motion attenuation relations of small and moderate earthquakes in Sichuan region*
}

\author{
Lanchi Kang $^{1, *}$ and Xing Jin ${ }^{1,2}$ \\ ${ }^{1}$ Fuzhou University, Fuzhou 350003, China \\ ${ }^{2}$ Earthquake Administration of Fujian Province, Fuzhou 350003, China
}

\begin{abstract}
On the basis of 10935 broadband velocity records of 135 earthquakes $\left(M_{\mathrm{L}} 3.0-6.4\right.$ and epicentral distance of 26-623 km) occurred from May 12 to June 10 in 2008, which are collected from 27 bedrock stations included in Sichuan Earthquake Monitoring Network, the corresponding acceleration records are obtained by a real-time simulation method. Then by regression analysis on the data, the relation between the peak ground acceleration and velocity attenuation of small and moderate bedrock earthquakes occurred in Sichuan region is acquired. And the relation is verified by a M4.8 earthquake took place recently in Wenchuan. Finally, the attenuation relations, which are coincident to the geological conditions in Sichuan region, are proposed by studying the records from Sichuan earthquake network.
\end{abstract}

Key words: real-time simulation; peak ground acceleration; peak ground velocity; regression analysis; attenuation relation

CLC number: P315.9 Document code: A

\section{Introduction}

On May 12, 2008, the great Wenchuan M8.0 earthquake occurred, which was felt even in Beijing, Shanghai, Guizhou, Chongqing, Hubei, and Jiangxi provinces and municipalities. The strong earthquake caused 70 thousand victims, 300 thousand injurers, nearly 20 thousand missing persons and enormous economic losses. The Wenchuan earthquake is one of the most destructive, most widely spreading, and the most difficultly rescuing events occurred in China since 1949. Almost 4.5 million houses collapsed and more than 10 million people became homeless in the earthquake. The seriously destructive area is about 100 thousand square kilometers. And the injury to the health and psychology of the people is hardly estimable.

After the main shock, almost 13550 aftershocks occurred to 12:00 of June 23, 2008 provided by the China Earthquake Networks Center. Among them, 187 events were M4.0-4.9 shocks, 28 events were M5.0-5.9 shocks, and five events were M6.0-6.4 shocks. The largest aftershock with M6.4 happened on May 25. The

\footnotetext{
* Received 4 February 2009; accepted in revised form 5 March 2009; published 10 June 2009.

• Corresponding author. e-mail: kanglanchi@yahoo.com.cn
}

information of aftershocks recorded by the Sichuan earthquake network has greatly enriched the seismic observation database, which can be used to execute interrelated research rapidly, so as to provide first-hand information to the work of earthquake prevention and disaster reduction in the disaster areas. In our current research on ground motion attenuation, the curves approximately obtained from the United States by a conversion method (Hu and Zhang, 1984) are still used for the lack of strong motion records in China. Now we can draw our own attenuation relations from these records by a method of statistical regression, rather than depending on the United States.

The ground motion attenuation relation is a method to estimate ground motion parameters, which can be used to provide relevant data for the post-disaster reconstruction. In Wenchuan earthquake, Sichuan earthquake network has recorded a large number of observation data, which are quite convenient for us to draw an attenuation law. In this paper, we just use these records to make a research on the attenuation law, in which the velocity records are simulated to the acceleration through the real-time simulation methods proposed by Jin et al (2004a, b). Then by a statistical analysis on these data, the attenuation law of peak ground acceleration 
(PGA) and peak ground velocity (PGV) has been acquired for the small and moderate earthquakes in Sichuan region.

\section{Data collection and collation analysis}

Nowadays, the earthquake networks in China have developed rapidly through the digitization reforms in the ninth Five-year Plan and the tenth Five-year Plan. And Sichuan Earthquake Monitoring Network has been equipped with a new kind of wide-banded, large dynamic, high-resolving velocity-type seismometer. The north-south seismic belt in China is an intensive earthquake zone stretching thousands of kilometers from Kunming in the south, to Chengdu and Lanzhou, and then to Yinchuan in the north. It covers the regions of Ningxia, eastern Gansu, western Sichuan and Yunnan. The Longmenshan belt is the most active segment lo- cated at the junction of Qinghai-Tibet plateau and Sichuan basin, and Sichuan is one of the active seismic areas in China. After the great Wenchuan earthquake, many networks had a failure in normal operation due to catastrophic damage. Therefore, 8505 broadband velocity records, with a magnitude range of M3.0-6.4 and an epicenter distance of 26-462 km, are selected from 105 earthquakes occurred from May 12 to June 10, 2008 recorded at 27 bedrock stations. In regression, the records in the two horizontal directions are regarded as two different ones. Among these 27 bedrock stations, 24 stations are equipped with CMG-3ESPC broadband seismometer with a band range of $60 \mathrm{~s}-50 \mathrm{~Hz}$; the other three are equipped with BBVS-60-type broadband seismometer with a band range of $60 \mathrm{~s}-50 \mathrm{~Hz}$. The distributions of magnitude and distance are listed in Table 1 and the locations of earthquake epicenters are shown in Figure 1.

Table 1 Number of seismic records in certain ranges of magnitude and epicenter distance

\begin{tabular}{|c|c|c|c|c|c|c|c|}
\hline \multirow{2}{*}{$\begin{array}{l}\text { Epicentral distance } \\
\qquad / \mathrm{km}\end{array}$} & \multicolumn{7}{|c|}{ Magnitude } \\
\hline & $3.0-3.4$ & $3.5-3.9$ & $4.0-4.4$ & $4.5-4.9$ & $5.0-5.4$ & $5.5-5.9$ & $>6.0$ \\
\hline $0-50$ & 18 & 5 & 15 & 27 & 20 & 6 & 0 \\
\hline $50-100$ & 56 & 49 & 75 & 186 & 138 & 39 & 11 \\
\hline $100-200$ & 139 & 97 & 194 & 354 & 266 & 67 & 22 \\
\hline $200-300$ & 130 & 51 & 152 & 260 & 185 & 34 & 16 \\
\hline $300-400$ & 96 & 44 & 125 & 188 & 147 & 22 & 18 \\
\hline$>400$ & 0 & 0 & 87 & 143 & 108 & 17 & 14 \\
\hline
\end{tabular}

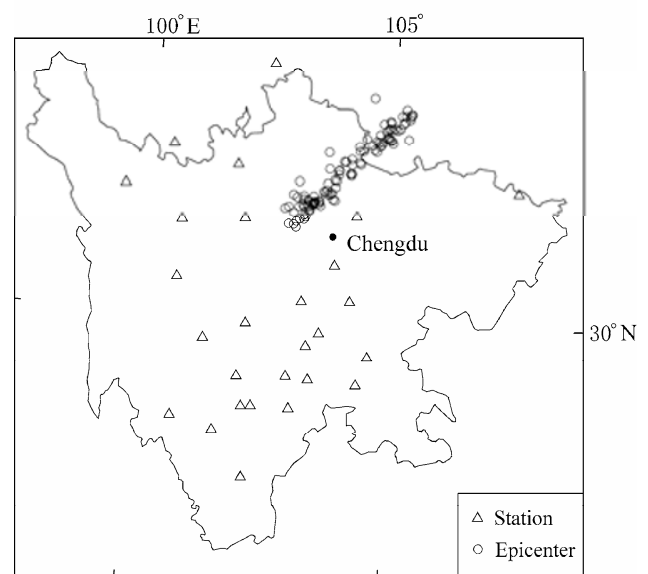

Figure 1 Epicentral distribution of earthquakes in this study.

In this paper, the real-time simulation method proposed by Jin et al (2004b) is used to obtain the corresponding acceleration. As a result, a new attenuation relation of PGA and PGV for the small to moderate earthquakes on the rock site in Sichuan region is established.

\section{Regression model and method of earthquake parameters}

The least-squares method is often used to determine the coefficients in the ground motion attenuation relation model. Many others are still the ways on this basis with certain improvements according to the characteristics of data distribution, such as the weighted least squares, consistent weighted least squares (Huo, 1989). Joyner and Boore (1981) put forward a two-step method to decouple the distance and magnitude. The first step is to regress distance and the second step is for magnitude, which can reduce their correlation impacts on the regression results. In this paper, the two-step method is used to determine the coefficients in the ground motion attenuation relation model: the first step is to fit the distance by the least-squares method; the second step is to fit the magnitude by the same method (Jin et al, 2008, Wang, 2001).

First of all, considering the impact of epicentral distance on the peak ground motion under the same magnitude, we select the model as follows: 


$$
\ln Y=a_{0}+b_{0} \ln \left(\Delta+R_{0}\right),
$$

where $a_{0}$ and $b_{0}$ are the functions of magnitude, respectively. The geometric diffusion term $R_{0}$ is taken as 10 $\mathrm{km}$ considering the focal depth in Sichuan region is generally about $10 \mathrm{~km}$; at the same time, considering that $a_{0}$ and $b_{0}$ have a linear relation with the magnitude
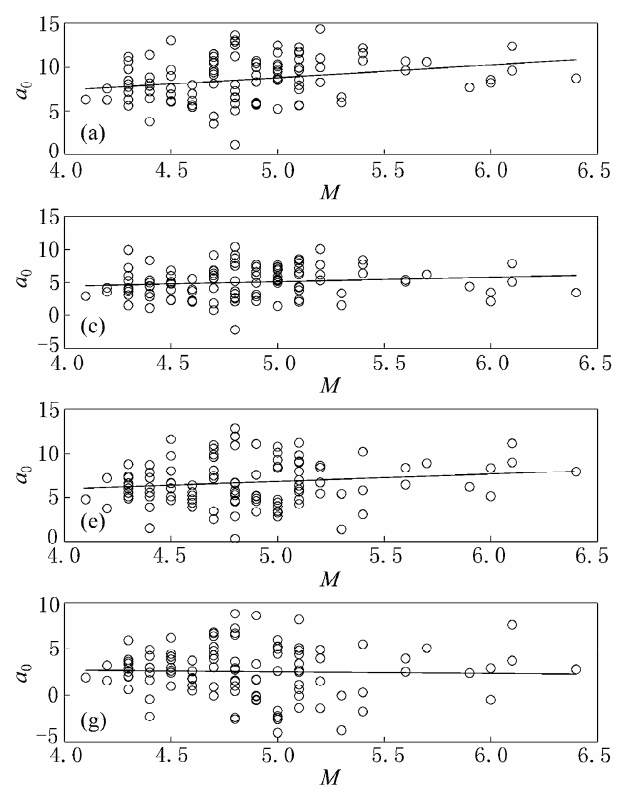

(see Figure 2), we select the following models:

$$
\left\{\begin{array}{l}
a_{0}=a+b M \\
b_{0}=c+d M
\end{array} .\right.
$$

Finally, the attenuation model is

$$
\ln Y=a+b M+(c+d M) \ln (\Delta+10) .
$$
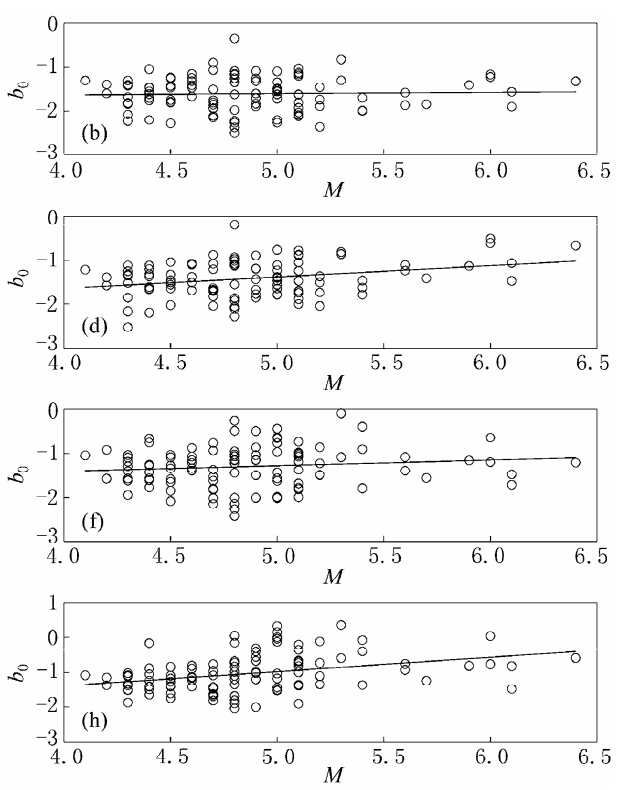

Figure 2 Relation of values $a_{0}$ and $b_{0}$ to magnitude by using horizontal acceleration (a, b), horizontal velocity $(c, d)$, vertical acceleration $(e, f)$ and vertical velocity $(g, h)$

\section{Regression results}

According to what mentioned in the section 3, the horizontal and vertical peaks are regressed in both acceleration and velocity records, respectively. Then the attenuation law of peak ground motion on bedrock sites in Sichuan region is acquired. The detail is described in Table 2.

Table 2 Regression coefficients of the attenuation law of peak ground motion on bedrock sites in Sichuan region

\begin{tabular}{cccccc}
\hline Model & $\begin{array}{c}\text { Ground-motion } \\
\text { parameter }\end{array}$ & $a$ & $b$ & $c$ & $d$ \\
\hline \multirow{4}{*}{ Two-step } & PGA-H & -5.4193 & 2.8426 & 0.68043 & -0.45477 \\
model & PGA-V & -3.1243 & 1.9749 & -0.2571 & -0.30687 \\
& PGV-H & -9.2058 & 2.8568 & 0.22338 & -0.32063 \\
& PGV-V & -6.3949 & 1.788 & -0.3158 & -0.13331 \\
\hline
\end{tabular}

Note: PGA-H and PGA-V denote horizontal and vertical PGA, respectively; and PGV-H and PGV-V denote horizontal and vertical PGV, respectively.

The peak ground acceleration and velocity curves are given with the magnitudes of 3.5, 4.0, 4.5, 5.0, 5.5 and 6.0, respectively and their changes along with epicentral distance $\Delta$. Figure 3 provides the peak accelera- tion and peak velocity attenuation curves in contrast with the original results, from which we draw the following results.

1) The calculated curves are basically consistent with the original records, which could reflect the attenuation trend of the original data. It can be seen from the regression results that the coefficients of magnitude term are all positive, which demonstrates that the peaks acceleration and velocity increase with magnitude enlarging; in addition, the coefficients of distance term and logarithmic distance term are negative, which indicates that the peak value decreases with the increasing distance.

2) The far-field curve is well fitting with the original data, while the near field fitting results have a bias. The reason for the bias might be the small amount of near field records, which result in a conservative estimation. We therefore conclude that this bias can be corrected in the future by collecting more data.

3) The attenuation speed of the vertical acceleration is slightly faster than that of the horizontal one under the same magnitude, which is consistent to the general 

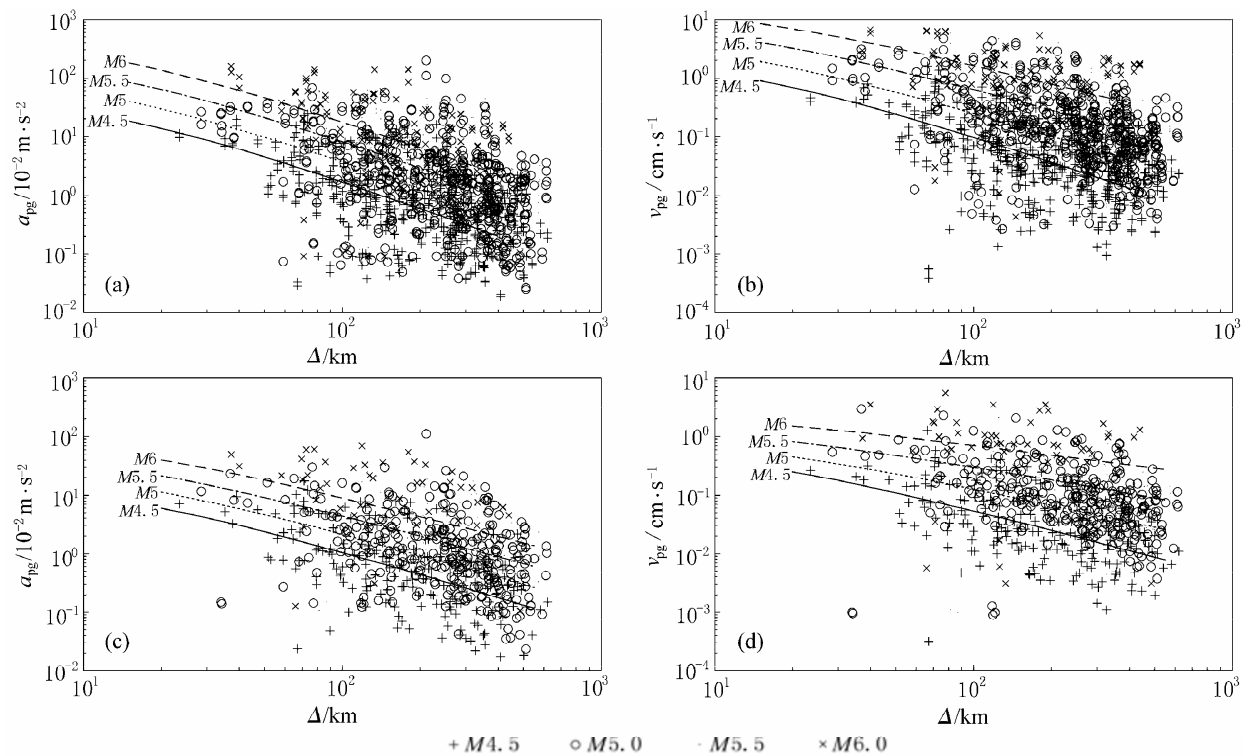

Figure 3 Comparison between the fitting results based on the two-step model and original records. (a) Horizontal acceleration; (b) Horizontal velocity; (c) Vertical acceleration; (d) Vertical velocity.

phenomenon that the high frequency components of vertical records attenuate faster.

\section{Comparison with other attenuation relations}

As the majority data of small-moderate earthquakes used in this paper were medium to far field records, so the comparison is made to the results selected from similar and representative researches conducted in the recent years. For example, the attenuation relations of Liu and Tsai (2005) and Bragato and Slejko (2005), which are briefly introduced as follows.

1) The attenuation relationship of Liu and Tsai (2005) including the attenuation relations of peak acceleration and velocity. The records used are from bedrock, hard-soil and soft-soil shallow earthquakes occurred in Taiwan from the year of 1993 to 2002 with a magnitude range of 4.0-7.1 and a distances less than $275 \mathrm{~km}$. The formula is

$$
\ln Y=a \ln (R+h)+b R+c M_{\mathrm{W}}+d,
$$

where $M_{\mathrm{W}}$ is the moment magnitude, $R$ is the hypocentral distance, and $h$ is a saturation coefficient of close earthquakes.

2) The attenuation relationship of Bragato and Slejko (2005) including the attenuation relations of peak and acceleration response spectra. The records used are bedrock and hard-soil shallow earthquakes in the eastern Alps happened from 1993 to 2002 with a magnitude range of 2.5-6.3 and a distance less than $134 \mathrm{~km}$. The formula is

$$
\lg Y=a+(b+c M) M+\left(d+e M^{3}\right) \lg \Delta,
$$

where $M$ is local magnitude and $\Delta$ is epicentral distance.

Figure 4 shows the curves of these three models with different magnitudes. It is apparent from the figure that the curves of this paper are very similar to Liu and Tsai's attenuation relation in near field. The difference not only in velocity but also in acceleration increases obviously with magnitude and distance increasing. Apart from the geographical differences, it is mainly due to the statistical data in different distributions and by different regression methods. Liu and Tsai's attenuation relations affect the attenuation of small earthquakes by using more earthquake records with great magnitudes. As a result, the value is larger for small earthquakes. Bragato and Slejko (2005) combined the bedrock and soil data together to make statistics directly by a multi-variant method. Because of more small earthquake records in near field are included in the statistics, the regression results in the near field should be close to the original records; while the attenuation law of the far-field strong earthquakes is affected, resulting in the estimated results for the far-field earthquakes are smaller.

At the same time, the results from this paper are compared with those of Huo (1989), too (see Figure 5). The comparison shows that, in the near field with low magnitude, the results of this paper are smaller; in the 

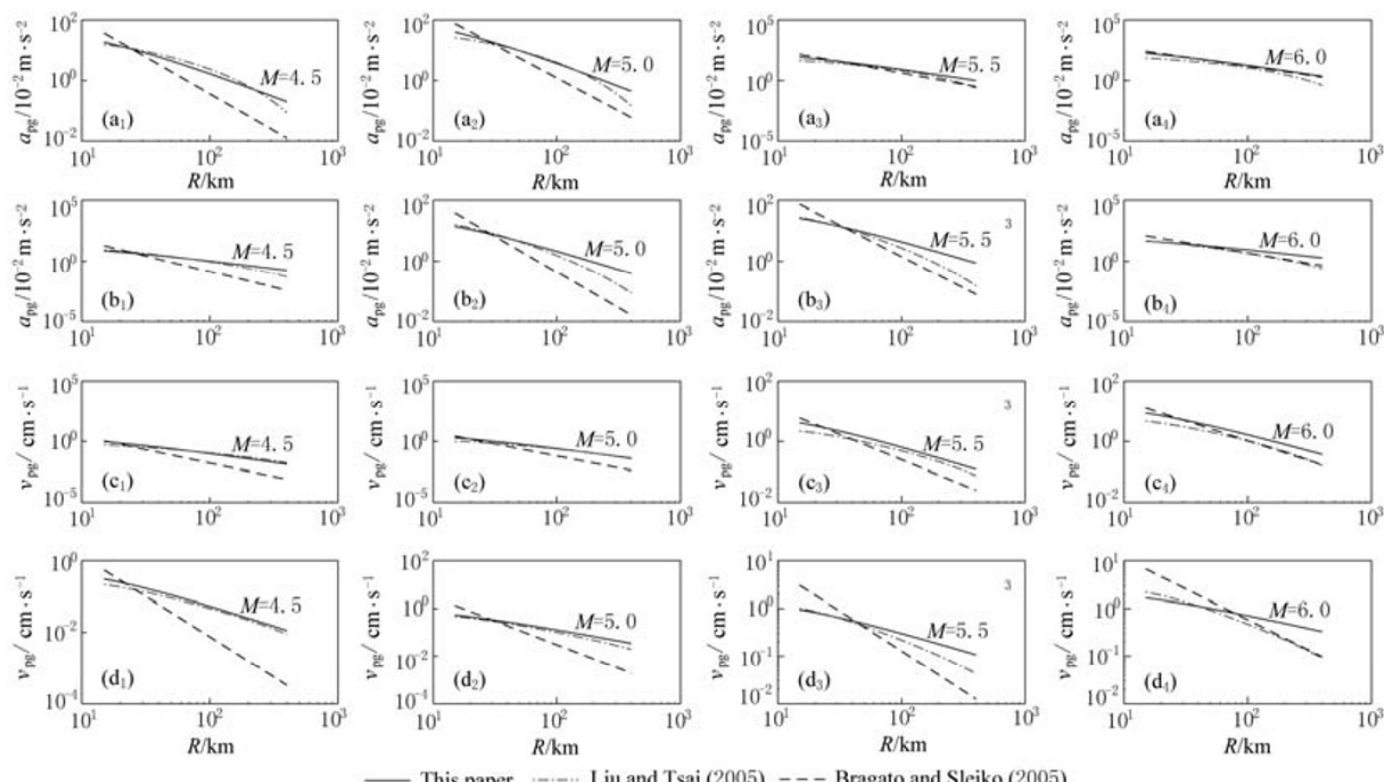

Figure 4 Comparison between the results in this paper and other results. (a) Horizontal acceleration; (b) Vertical acceleration; (c) Horizontal velocity; (d) Vertical velocity.

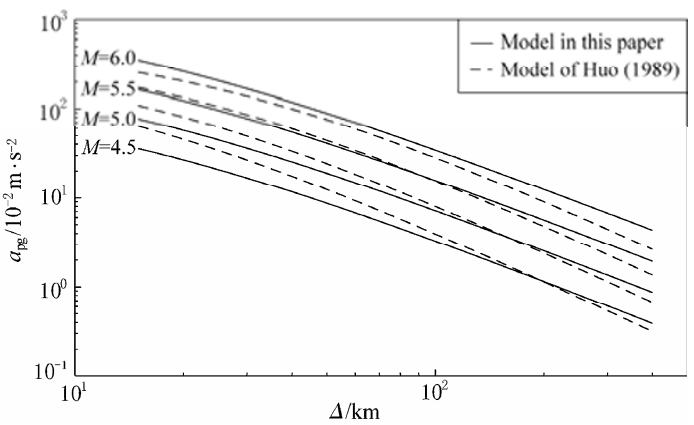

Figure 5 Comparison between acceleration of this paper and Huo (1989).

moderate distance, the two results are approximate; and in the far distance, the results in the paper are larger. Considering as a whole, the result of this paper is greater than that of Huo (1989) with magnitude increasing, and the attenuation speed in this paper is faster than that of Huo (1989). The reasons could be due to the differences in the geological condition, model, and regression method, etc.

\section{Verification to attenuation relation- ship}

At 13:51 on June 17, 2008, a M4.8 earthquake occurred in Ningqiang, Shaanxi province. The velocity records from this earthquake are simulated to acceleration with the same frequency bands ranging from 0.05
$\mathrm{Hz}$ to $20 \mathrm{~Hz}$ by using real-time simulation methods proposed by Jin et al (2003). The corresponding acceleration records are obtained and its peak ground velocity and acceleration are extracted in order to verify the results in the present paper (see Figure 6).

It is evident that the attenuation curve of this paper is basically consistent with the original data, which shows the results of this paper are of greatly practicality.

\section{Conclusions}

In this paper, the velocity records collected from Sichuan Earthquake Monitoring Network are simulated by the method of Jin et al (2004b) to obtain the acceleration records; then by taking a two-step model, the horizontal and vertical peak acceleration and velocity are regressed, respectively; finally, the attenuation laws for the peak ground motion of bedrock sites in Sichuan region are acquired. According to the study, the model is applicable on the conditions, that is: (1) epicentral distance is $26-462 \mathrm{~km}$, (2) magnitude is $M 3.0-6.4$, and (3) site condition is bedrock.

The regression results in this paper are compared with the current international ground motion attenuation relations for small and moderate earthquakes, and certain possible reasons for the existing differences are found. Then verification is made with the $M 4.8$ earthquake occurred on June 17, 2008. It is apparent from the results that the calculated attenuation relations are basically 

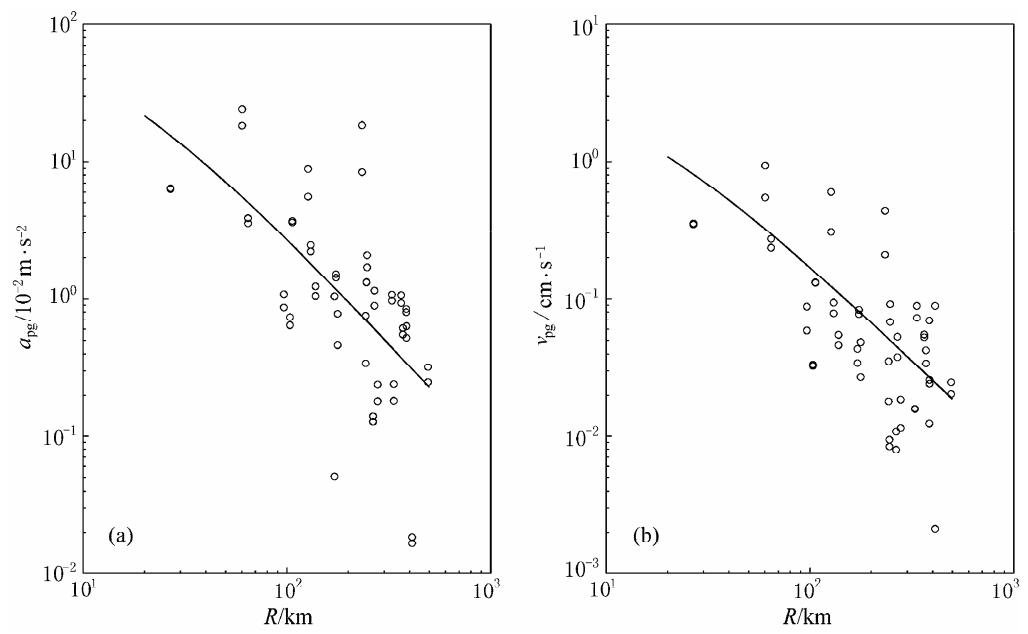

Figure 6 Comparison between acceleration (a) and velocity (b) of this paper and original records (denoted by circle).

consistent with the original records. We therefore can draw a conclusion that the results of this paper have a better practicality.

During a long period, our research on attenuation relation depends mainly on the conversion method adopted by the United States due to lack of strong motion records in China. After the 2008 Wenchuan earthquake, a large number of M5-6 aftershocks occurred in Sichuan region. And on the basis of these data, we have drawn our own attenuation law. Therefore, what we have done in the current stage have laid the foundation for the attenuation relation of small and moderate earthquakes expanding to that of strong ground motion in the future. At present, only the peak acceleration and velocity of statistical regression are provided. As to other ground motion parameters, we will make an introduction in another paper.

Acknowledgements Heartfelt gratitude is given to China Earthquake Networks Center for providing valuable data.

\section{References}

Bragato P L and Slejko D (2005). Empirical ground-motion attenuation relations for the Eastern Alps in the magnitude range 2.5-6.3. Bull Seism Soc Amer 95(1): 252-276.
Hu Y X and Zhang M Z (1984). A method of predicting ground motion parameters for regions with poor ground motion data. Earthquake Engineering and Engineering Vibration 4(1): 1-11 (in Chinese with English abstract).

Huo J R (1989). Study on the Attenuation Laws of Strong Earthquake Ground Motion Near the Source. Ph D Dissertation, Institute of Engineering Mechanics, State Seismological Bureau, Harbin, China, 29-39 (in Chinese with English abstract).

Jin X, Ma Q and Li S Y (2003). Comparison of four numerical methods for calculating seismic dynamic response of SDOF system. Earthquake Engineering and Engineering Vibration 23(1): 18-30 (in Chinese with English abstract).

Jin X, Ma Q and Li S (2004a). Real-time simulation of ground velocity using digital accelerograph record. Earthquake Engineering and Engineering Vibration 24(1): 49-84 (in Chinese with English abstract).

Jin X, Ma Q and Li S (2004b). Real-time simulation of ground displacement and acceleration using digital velocity record. Earthquake Engineering and Engineering Vibration 24(6): 9-14 (in Chinese with English abstract).

Jin X, Kang L C and Ou Y P (2008). Ground motion attenuation relation for small to moderate earthquakes in Fujian region, China. Acta Seismologica Sinica 21(3): 283-295.

Joyner W and Boore D (1981). Peak horizontal acceleration and velocity from strong motion records including records from the 1979 Imperial Valley, California, earthquake. Bull Seism Soc Amer 71(3): 2 011-2 038.

Liu K S and Tsai Yi-Ben (2005). Attenuation relation of peak ground acceleration and velocity for crustal earthquake in Taiwan. Bull Seism Soc Amer 95(3): $1043-1058$.

Wang G X (2001). Strong Ground Motion Attenuation. Ph D Dissertation. Institute of Engineering Mechanics, State Seismological Bureau, Harbin, China, 23-29 (in Chinese with English abstract). 